\title{
The effect of serial founder-flush cycles on quantitative genetic variation in the housefly
}

\author{
EDWIN H. BRYANT \& LISA M. MEFFERT \\ Department of Biology, University of Houston, Houston, TX, U.S.A.
}

\begin{abstract}
Experimental populations of the housefly, initiated from a single outbred natural population, were subjected to five serial founder events of one, four, or 16 pairs of flies. After each bottleneck the populations were allowed to flush to approximately 1000 pairs, at which time they were assayed for additive genetic variance for eight morphometric traits using parental-offspring covariances. Additive genetic variance for all bottleneck sizes rose above the level of the outbred control in response to the first bottleneck and remained comparable to or higher than that of the control over most of the successive bottleneck episodes; no bottleneck size exhibited additive genetic variance significantly below the level of the control throughout the experiment. Such changes in additive genetic variance in response to bottlenecks are inconsistent with a model of neutral additive gene action, suggesting that non-additive components of genetic variance are likely to have affected the traits. Two models of non-additive genetic variance, pure dominance and additive-by-additive epistasis, provided reasonable fits to our data, but were not distinguishable from each other. Both empirical and theoretical results suggest that additive genetic variance for quantitative traits can remain high despite repeated reduction of the population size to as low as a single mated pair. Historical bottlenecks cannot be accurately inferred from levels of additive genetic variance for complex quantitative characters that are affected by non-additive components of genetic variance.
\end{abstract}

Keywords: bottlenecks, founder events, housefly, quantitative genetics.

\section{Introduction}

When the effects of individual loci affecting a quantitative trait combine in complete additivity, a population bottleneck is expected to decrease the genetic variance associated with the trait (Wright, 1951; Lande, 1980; Falconer, 1989). However, when the separate effects of individual alleles do not act in such a purely additive fashion, a population bottleneck may not lower additive genetic variance. For example, it has been shown theoretically that additive genetic variance can increase after a population bottleneck in the case of dominance among alleles within loci, even though the loci affecting the trait combine additively (Robertson, 1952; Cockerham \& Tachida, 1988; Tachida \& Cockerham, 1989a; Jiang \& Cockerham, 1990). Moreover, theory has shown that interaction among individual loci (i.e. epistasis) can also result in increased additive genetic variance as the bottleneck causes a portion of the nonadditive variance to be converted to the additive component (Cockerham \& Tachida, 1988; Goodnight, 1987, 1988; Tachida \& Cockerham, 1989b).

Correspondence: E. H. Bryant.
The degree to which quantitative traits are affected by non-additive genetic processes is largely unknown. The widespread occurrence of inbreeding depression implicates directional dominance for viability and reproductive traits (Robertson, 1955; Crow \& Kimura, 1970; Charlesworth \& Charlesworth, 1987; Falconer, 1989); however, evidence for epistasis affecting quantitative traits is less compelling (see reviews by Barker, 1974, 1979; Barton \& Turelli, 1989; Cohan et al., 1989; Geiger, 1988), although the theoretical likelihood of epistasis is high for fitness traits under stabilizing selection (Robertson, 1955; Wright, 1965, 1969, 1977). When epistatic interactions are found, they are often not large relative to the additive genetic component (e.g. Kearsey \& Kojima, 1967; Shrimpton \& Robertson, 1988a,b; Barnes et al. 1989; Paterson et al., 1990).

One approach to detecting non-additive effects is to subject experimental populations to inbreeding and/or bottleneck regimes to estimate changes in the additive component of variance (or heritability) relative to neutral expectation. Several cases with apparent increases in the additive genetic variance of inbred and/or bottleneck lines have been reported. Lopez- 
Fanjul \& Villaverde (1989) found an average increase in the genetic variance associated with viability within and among lines of $D$. melanogaster subjected to fullsib mating. Abplanalp (1988) suggested that nonadditive effects were responsible for increased genetic variance of egg production within inbred lines of white leghorn chickens. Increased heritability for sternopleural bristle number was reported by Lints \& Bourgois (1982) for a line of D. melanogaster that had undergone an accidental bottleneck, and Carson \& Wisotzkey (1989) found increased karyotypic variance in a population of $D$. silvestris that had been reduced to six individuals. Polans \& Allard (1989) reported increased coefficients of (phenotypic) variance for five life-history traits in the ryegrass, Lolium multiflorum, which had been subjected to experimental bottlenecks.

In an experiment to investigate the effects of experimental founder-flush of three sizes (one, four, and 16 pairs) in the housefly, we observed increases in the additive component of variance for eight morphological traits estimated from parental-offspring covariances (Bryant et al., 1986). Moreover, all 12 bottleneck lines had higher average additive genetic variance relative to neutral expectation $\left(\chi^{2}\right.$ for $1: 1$ expectation around neutral expectation $=12.0$; $P<0.001$ ), providing clear evidence that additive genetic variance for quantitative traits can increase following a single founder-flush cycle. Of these experimental lines, two lines for each bottleneck size were carried through four additional founder-flush cycles with assays for additive genetic variance after each flush phase. Because the minimum level of inbreeding after five serial bottlenecks is a 10 -fold difference between the single-pair and 16-pair lines, these data should provide a more powerful assessment of the effect of inbreeding on additive genetic variance than did the single bottleneck episode evaluated previously. Specifically, we document changes in additive genetic variance over the course of five serial bottlenecks and compare the observed changes in additive genetic variance with patterns predicted from models of additive, dominance, and epistatic genetic variance. Patterns of divergence in phenotypic means among lines are evaluated in a separate paper (E. H. Bryant \& L. M. Meffert, unpublished observations). These are the first such empirical data investigating the effects of multiple bottlenecks on quantitative genetic variation.

\section{Materials and methods}

A single large sample of an outbred population of houseflies was taken from a landfill population at Alvin, TX. After establishment in the laboratory, bottleneck lines were initiated using the offspring from either one, four, or 16 pairs of flies. By combining progeny from isolated male-female pairs, the number of founders was exactly determined. The bottleneck populations were allowed to flush to normal laboratory size (approximately 1000 pairs), taking about five generations. Four replicate lines were established for each bottleneck size for the first bottleneck, and two of these lines per bottleneck size treatment were subjected to an additional four founder-flush cycles, giving a total of five serial bottleneck episodes per line. The control (base population) line was maintained in the laboratory throughout the course of the experiment at approximately 1000 pairs.

After each population flush, additive genetic variances for eight morphometric traits in the bottleneck and control lines were separately estimated as the average of sire, dam, and midparental-offspring covariance of log-transformed measurements taken with an ocular micrometer. Negative variance estimates were set to zero. As these negative values occurred more often in the control, due to it having the lowest genetic variance across the experiment, tests of differences between bottleneck lines and control are conservative. The eight morphometric traits were (for a full description of traits, see Bryant, 1977): wing length, wing width, head width, inner-eye separation, scutellum length, scutellum width, thoracic suture length, and metafemur length. The separate estimates of additive genetic variance for the eight traits were averaged over traits within each line and bottleneck episode. The estimates for the two replicate lines within a bottleneck size were then averaged, weighting each by the number of families used to obtain the estimate, to obtain a single estimate of additive genetic variance for each bottleneck size treatment over the five cycles. The parents and three female offspring were measured per family; the average numbers of families used for each replicate line and bottleneck episode were 28, 29, 35, and 36 for the single-pair, four-pair, 16-pair, and control, respectively. All flies for quantitative genetic variation estimates were reared under optimal densities of $80 \mathrm{eggs} / 18 \mathrm{~g} \mathrm{CSMA}$ larval medium at $27^{\circ} \mathrm{C}$ to control for the effects of larval density and temperature on size. For convenience all resulting variance estimates were multiplied by $10^{4}$.

\section{Results}

\section{Quantitative genetic estimates}

In order to compare additive genetic variances for bottleneck and control lines over the serial bottlenecks the control line was first examined for possible monotonic changes through the duration of the experi- 
ment. The six successive estimates for the average additive genetic variance for the control and the linear regression of these values onto bottleneck episode number are shown in Fig. 1. Average additive genetic variance fcr the control was significantly greater than zero $(P<0.05)$ but showed no significant linear change over the 33 generations of the experiment $(P>0.05)$. As there was no linear trend in the control, and the number of families was approximately the same among lines, the separate estimates over the six sampling periods can then be combined into a single mean (and variance) estimate for the control, to be compared with the corresponding estimates for the bottleneck treatments; this procedure is essentially equivalent to an experimental bootstrap (e.g. see Efron, 1982; Efron \& Gong, 1983).

The estimates for the additive genetic variances averaged over the two replicate lines for each bottleneck size and the expected decline in variability, based on purely additive effects [i.e. a loss of $1 /(2 n)$ per bottleneck episode from the average control value, where $n$ is the number of founders], are given in Fig. 2, along with the 95 per cent upper confidence bounds to this neutral expectation. All three bottleneck sizes exhibited levels of additive genetic variance above the control and significantly greater than neutral expectation for at least some of the bottleneck episodes ( 5 of 5 for the single-pair; 2 of 5 for the four-pair; 4 of 5 for the 16-pair, Fig. 2). Additionally, no bottleneck line had significantly lower additive genetic variance than the control during the entire course of the experiment (lower 95 per cent confidence limit for additive genetic variance $=2.39$ ). Hence, not only were the additive genetic variances for bottleneck lines after the first bottleneck episode higher than predicted from neutral theory, as we previously reported, but they remained comparable to or higher than the control throughout the additional four founder-flush cycles.

\section{Effects of non-additive components of variance}

The neutral additive model appears inadequate to explain our results, so models incorporating nonadditive components of variance are warranted. The effect of directional dominance on additive genetic variance after a single bottleneck was addressed in a prior study (Bryant et al., 1986), and we extend the model here to include serial bottlenecks. Briefly, we assume that the loci affecting a trait act independently (i.e. exhibit no epistasis), each with complete dominance between two alleles. The additive genetic variance for the $i$ th locus is then $V_{\mathrm{A}}(i)=8 p q^{3} a^{2}$, where $q$ is the frequency of the recessive allele $(p=1-q)$ and $a$ is the average effect of an allelic substitution on the trait (Falconer, 1989). The expected additive genetic variance contributed by a locus is the average of the additive genetic variances for all possible allele frequencies, weighted by their binomial probabilities of occurrence after a bottleneck, so that $V_{\mathrm{A}}=\Sigma V_{\mathrm{A}}(i) \times \operatorname{Pr}\left(p_{i}\right)$ is the probability of the recessive allele being $p_{i}$ based on binomial expansion. No further changes in allele frequencies are assumed through each flush phase, so each successive bottleneck is based on the allelic frequency distribution of the previous bottleneck. Assuming that the initial frequencies of recessive alleles at all loci affecting the trait are approximately equal, the distribution of interlocus effects is identical to the expected distribution of effects within a single locus, so $V_{\mathrm{A}}$ is also the expected additive genetic variance after a bottleneck averaged over all loci.
Fig. 1 Additive genetic variance (averaged over eight traits) for the outbred control over the six sampling periods of the experiment. Linear regression of additive genetic variances onto sampling time is shown, along with the average value for the control (horizontal line, average $=5.40$ ).

$y=4.3848+0.40764 x ; r=0.36$.

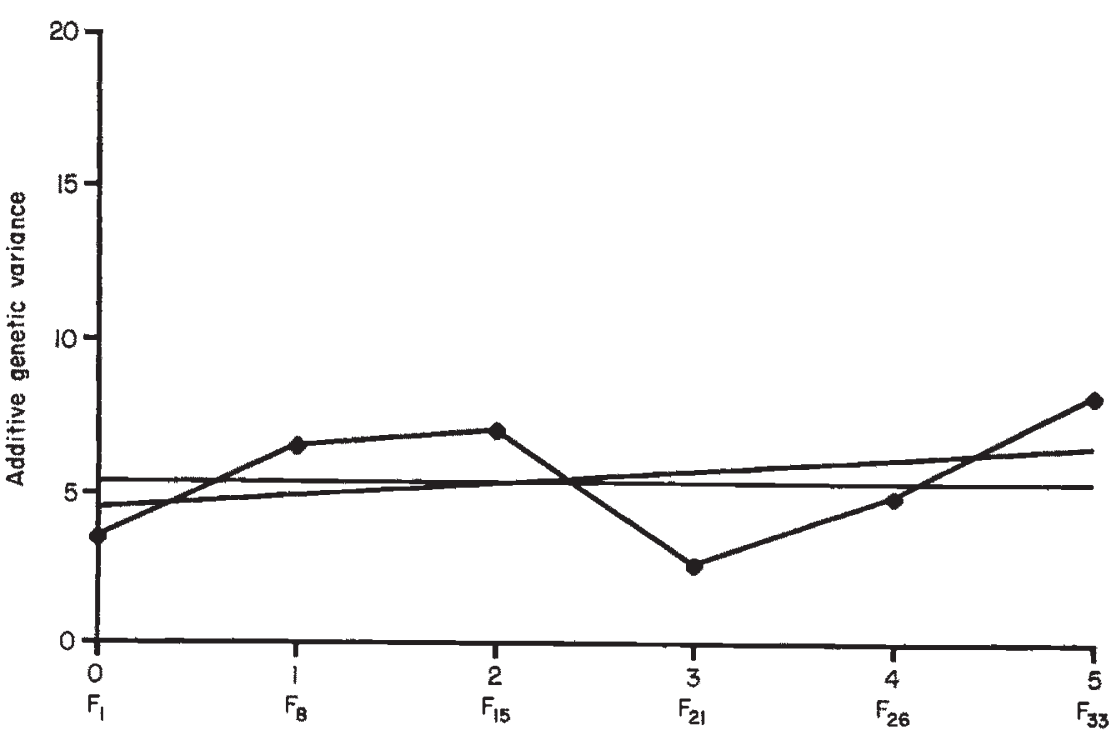



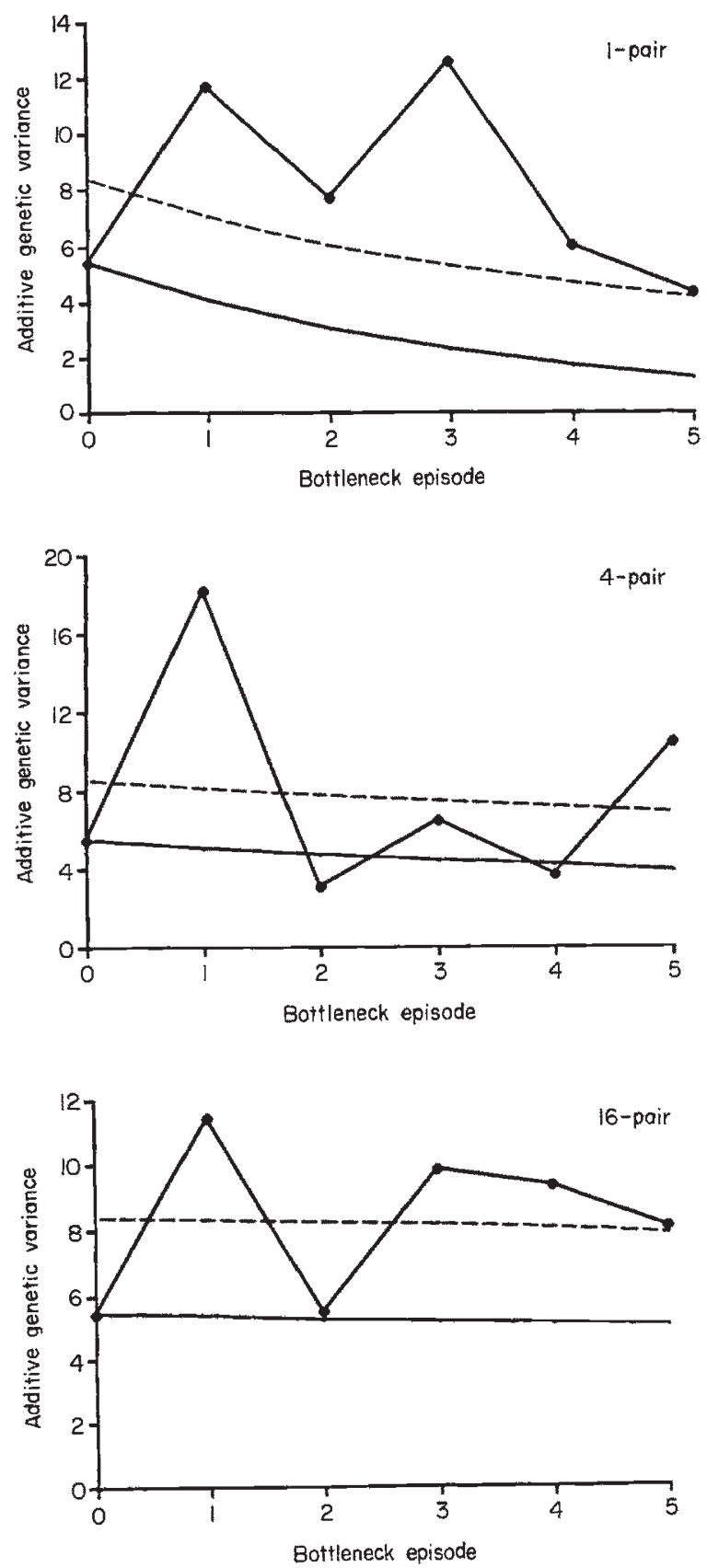

Fig. 2 Additive genetic variances for the three bottleneck sizes over the five bottleneck episodes. Expected values $(-)$ and 95 per cent upper confidence bounds $(---)$ under the neutral additive model are shown, based on average additive genetic variance of the control.

An approach to studying the effects of epistatic interaction on additive genetic variances in bottlenecked populations was provided by Goodnight (1987, 1988), using the co-ancestry and drift models developed by Cockerham (1967), Cockerham \& Weir (1973) and Weir \& Cockerham (1973). To simulate our estimates of additive genetic variance, taken after a period of population flush following each bottleneck episode, we used the rates of conversion of nonadditive to additive genetic variance for free recombination, as suggested by Goodnight (1988). For each successive bottleneck, the current level of additive genetic variance was decremented by the expected neutral rate of $1 /(2 n)$ and, as well, incremented by the conversion of non-additive to additive genetic variance, the net result of these two processes being the new value for additive genetic variance. The rates of conversion of non-additive to additive genetic variance during each bottleneck episode from Goodnight (1988 and personal communication) were 75.0 per cent, 23.4 per cent and 6.2 per cent for the single-, four-, and 16pair bottleneck sizes, respectively. Conversely, the percentage of the non-additive variance retained after each successive bottleneck was 56.25 per cent, 87.8 per cent and 96.97 per cent, for the single-, four-, and 16-pair lines, respectively.

Without selection, additive genetic variance in either non-additive model would increase with up to nearly 50 per cent inbreeding, depending upon the initial magnitude of dominance or epistatic variance in the ancestral population. To account for apparent declines in additive genetic variance with lower inbreeding levels, and to generalize the basic models, we incorporated selection into both non-additive models. For the dominance model we assumed selection against the recessive homozygote during the flush phase, so the decrease in frequency of the recessive allele at each locus was taken to be $\Delta q=-s q^{2} p$ per generation, where $s$ is the selection coefficient against homozygous recessives with frequency $q^{2}$ (Falconer, 1989). In the epistastic model, selection acted directly against the component of additive genetic variance as an overall percentage decrement in additive genetic variance during the entire flush phase. In both models, selection was applied for the five generations of each population flush following each successive bottleneck. The parameters varied were: initial frequency of recessive alleles (dominance model), the initial percentage of non-additive genetic variance (epistatic model) and the intensity of selection (both models). Expected changes in additive genetic variance were calculated for five serial founder-flush cycles, and a response surface was fitted to the resultant additive genetic variances using the distance-weighted least-squares smoothing methods of SYGRAPH (SYSTAT, Inc., Evanston, IL, U.S.A.).

Figures $3 a-3 c$ and $4 a-4 c$ show the response surfaces of additive genetic variance for the dominance model and the epistastic model, respectively, for the five bottleneck episodes and three bottleneck sizes. The two initial allele frequencies of $q=0.1$ (upper 

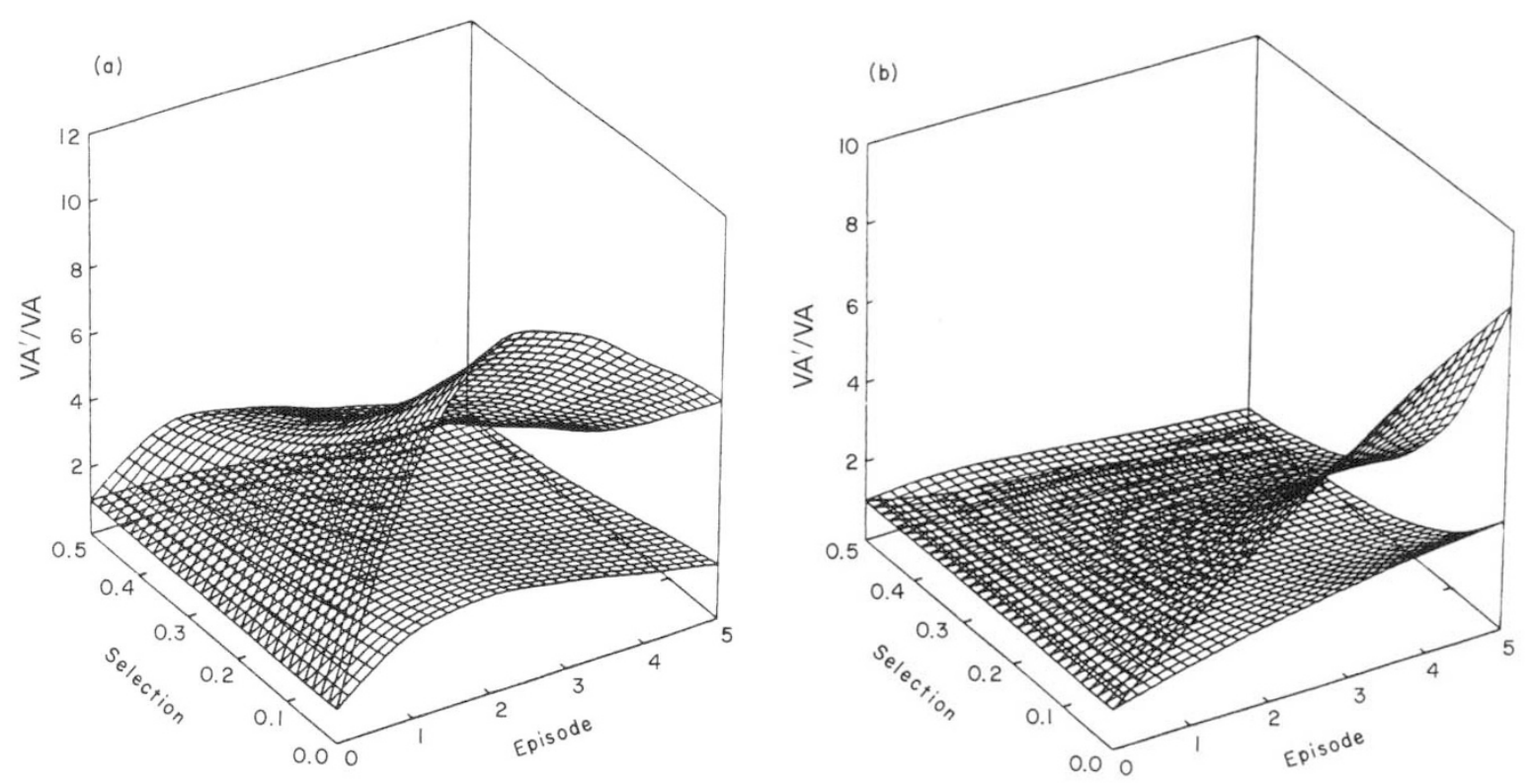

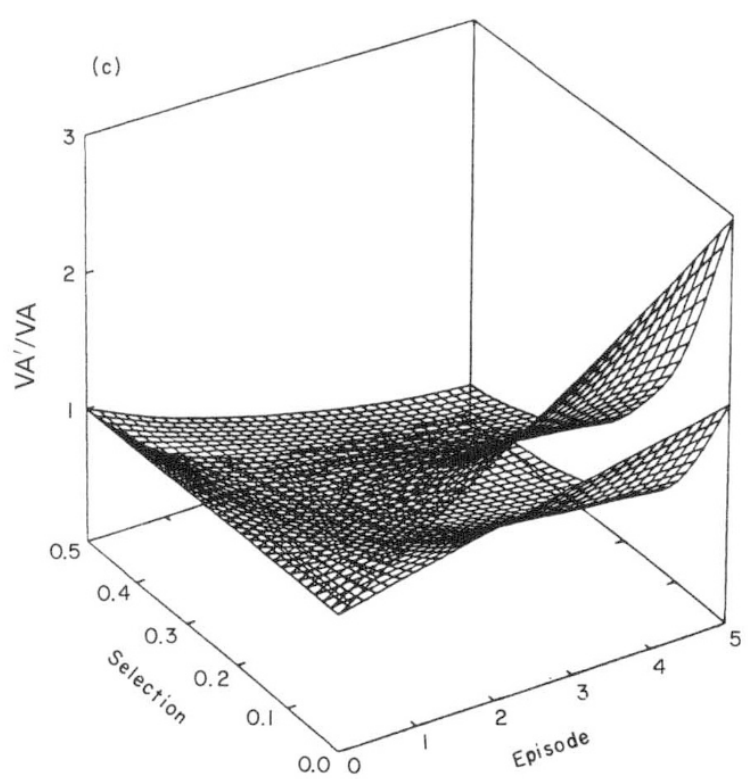

curve) and $q=0.2$ (lower curve) (dominance model) and initial levels of non-additive genetic variance of 75 per cent (upper curve) and 60 per cent (lower curve) (epistatic model), over a range of selection coefficients $(s=0.0$ to $s=0.5)$ are shown for each model. These parameter ranges were chosen from among a larger set modelled because the response surfaces bracketed the observed additive genetic variances. In the dominance model without selection, additive genetic variance increases for the single pair lines until the third bottleneck, while for the larger bottleneck sizes additive genetic variance increases through all five bottlenecks. With increasing selection, additive genetic variance for all three bottlenecks sizes initially rises and then begins to decrease in later bottlenecks. The epistatic model showed a very similar response in additive genetic variance for the three bottleneck sizes.

To identify which parameter conditions fit the data best, an overall least-squares fit of the observed additive genetic variances to those predicted by the models was computed for each set of parameters and summed over the three bottleneck sizes. The best fit for the dominance model occurred with an initial recessive allele frequency $(q)$ of 0.2 and selection $(s)$ of 0.1 , yielding a least-squares value of 11.37 . The best overall leastsquares fit of the data to the epistatic model occurred with an initial non-additive genetic variance of 60 per cent and no selection, yielding a least-squares value of 9.48 , which was slightly better than that for the dominance model. Both models, however, fitted the data 

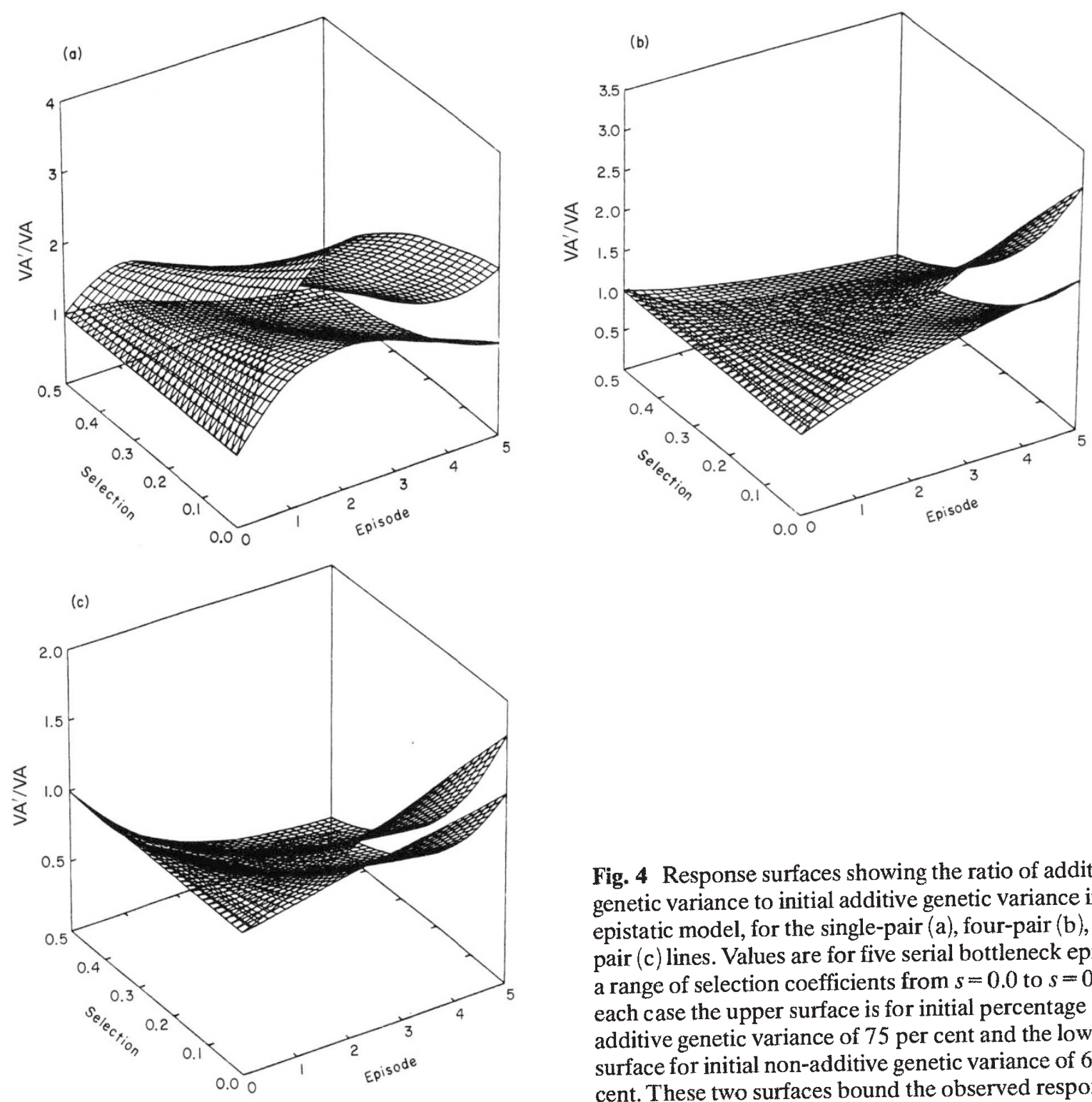

Fig. 4 Response surfaces showing the ratio of additive genetic variance to initial additive genetic variance in the epistatic model, for the single-pair (a), four-pair (b), and 16pair (c) lines. Values are for five serial bottleneck episodes for a range of selection coefficients from $s=0.0$ to $s=0.5$. In each case the upper surface is for initial percentage of nonadditive genetic variance of 75 per cent and the lower surface for initial non-additive genetic variance of 60 per cent. These two surfaces bound the observed responses.

considerably better than the neutral model, which yielded a least squares fit of 18.55 .

\section{Discussion}

Non-additive genetic processes must have structured our initial trait variances because the data clearly do not fit neutral additive expectation by exhibiting increased rather than decreased additive genetic variance following an initial bottleneck. As electrophoretic variation for these same lines largely conformed to neutral expectation by exhibiting continued losses in both the average heterozygosity and number of alleles over the five bottlenecks, in accordance with bottleneck size (McCommas \& Bryant, 1990), the

increased levels for additive genetic variances were not attributable to accidential inter-line contamination. Nor were the populations large enough for sustained periods of time (flushes averaged approximately five generations) to make mutation a likely source of new variation, even with the high mutation rate reported for quantitative traits (e.g. Lande, 1976; Lynch, 1985). The initial increase in additive genetic variance persisted for most lines despite the intervention of subsequent bottlenecks; therefore, the level of additive genetic variance for morphometric traits in relation to a known ancestor is a poor indicator of historical bottlenecks.

While the model of Goodnight (1988) incorporating additive-by-additive epistasis fitted our data slightly better than did the pure dominance model, it is clearly diffi- 
cult to differentiate between these possible effects, based on a least-squares fit alone. Our models only contrasted each non-additive genetic process in the absence of the other, and thus it would be far more difficult to dissect out separate processes if both dominance and epistasis affected the traits.

In testing for differences in additive genetic variance between bottleneck lines and the control, we employed the variance of repeated measures from the control. This is satisfactory as long as the variance of bottleneck lines was not greater than that of the control. Even though the effects of linkage are minimized by the flush (Avery \& Hill, 1977; Cockerham \& Tachida, 1988; Cockerham \& Weir, 1983), the variance among bottleneck lines could still be inflated by the initial sampling of founders (Lynch, 1988). Lynch (1988) predicted that the coefficients of variation among replicate lines after a single bottleneck, based on founder size alone, for the three bottleneck sizes of our protocol, would be 0.50 , 0.25 , and 0.12 , for the single-, four-, and 16-pair lines, respectively. In contrast, these coefficients of variation were $0.33,0.36$, and 0.29 , respectively. As the coefficient of variation from resampling the control line was 0.40 , every bottleneck treatment had a lower coefficient of variation than the control (none was significantly different from the control; $P>0.05$ ). Moreover, the coefficient of variation for the single-pair lines should be four times greater than that for the 16-pair lines, but the high congruence of among line coefficients of variation supports our basis for significance testing and suggests that neither linkage nor initial sampling of founders significantly affected our analyses. Because the near concordance of coefficients of variation among bottleneck treatments and control suggested that additive genetic variance may be lognormally distributed (Bryant, 1986), the tests in Fig. 2 were repeated using log-transformed additive genetic variances with nearly identical outcomes, so nonnormality of additive genetic variances did not apparently prejudice our analyses.

Because additive genetic variance for fitness-related traits is generally regarded as an indicator of evolutionary potential, at least in the short term (Fisher, 1958 ), bottlenecks have generally been assumed to lower the rate of evolutionary change (e.g. Barton \& Charlesworth, 1984; Charlesworth \& Smith, 1982). However, our bottleneck populations may not have sacrificed much of their evolutionary potential, even when subjected to repeated single-pair bottlenecks, in that they retained much of the additive genetic variance present in the outbred population. While our morphometric traits are not components of fitness per se, they are likely to be related to fitness through a correlation with general body size (e.g. Bryant et al., 1986), so sufficient additive genetic variance for components of fitness also may have remained in these bottleneck populations. This is supported by parallel data on fitness components for these same lines reported by Bryant et al. (1990) and Meffert \& Bryant (1991), who showed that by the end of the experiment most bottleneck lines were as fit as the outbred control despite being significantly lower in these fitness components after the first bottleneck (Bryant et al., 1986). The issue as to whether or not bottlenecks seriously erode evolutionary potential remains moot; consequently, inferences as to whether historical bottlenecks had occurred will not be accurate using genetic variances for quantitative traits.

\section{Acknowledgements}

This study was supported by grants from the National Science Foundation (BSR-8198128, BSR-8800977 and BSR-9106591) and The University of Houston Coastal Center. We thank Dr Charles Goodnight for reading and commenting on an earlier draft of this manuscript and Eddie Jazairi for technical assistance during the study.

\section{References}

ABPLANALP, H. 1988. Selection response in inbred lines of white leghorn chickens. In: Weir, B. S., Eisen, E. J, Goodman, M. M. and Namkoong (eds) Proceedings of the Second International Conference on Quantitative Genetics, Sinauer Associates, Sunderland, MA, pp. 360-378.

AVERY, P. J. AND HILL, W. G. 1977. Variability in genetic parameters among small populations. Genet. Res., 29, $193-213$

BARKER, J. F. S. 1974. The state of information concerning deviations from additivity of gene effects. In: Proceedings of the First World Congress on Genetics Applied to Livestock Production, Vol. 1, Gasri, Madrid, Spain, pp. 373-383.

BARKER, J. S. F. 1979. Inter-locus interactions: A review of experimental evidence. Theoret. Popul. Biol., 16. 323-346.

BARNES, P. T., HOLLAND, B. AND COURREgeS, v. 1989. Genotypeby-environment and epistastic interactions in Drosophila melanogaster: The effects of Gpdh allozymes, genetic background and rearing temperature on larval developmental time and viability. Genetics, 122, 859-868.

BARTON, N. H. AND CHARLESWORTH, B, 1984. Genetic revolutions, founder effects, and speciation. Ann. Rev. Ecol. Syst., 15, 133-164.

BARTON, N. H. AND TURELLI, M. 1989. Evolutionary quantitative genetics: How little do we know? Ann. Rev. Genet., 23, 337-370.

BRYANT, E. H. 1977. Morphometric adaptation of the housefly, Musca domestica L., in the United States. Evolution, 31, 580-596. 
BRYANT, E. H. 1986. On the use of logarithms to accommodate scale. Syst. Zool., 35, 552-559.

BRYANT, E. H., McCOMMAS, S. A. AND COMBS, L. M. 1986. The effect of an experimental bottleneck upon quantitative genetic variation in the housefly. Genetics, 114, 1191-1211.

BRYANT, E. H., MEFFERT, L. M. AND McCOMMAS, S. A. 1990. Fitness rebound in serially bottlenecked populations of the house fly. Am. Nat., 136, 542-549.

CARSON, H. L. AND WISOTZKEY, R. G. 1989. Increase in genetic variance following a population bottleneck. Am. Nat., 134, 668-673.

CHARLESWORTH, D. AND CHARLESWORTH, B. 1987. Inbreeding depression and its evolutionary consequences. Ann. Rev. Ecol. Syst., 18, 237-268.

CHARLESWORTH, B. AND SMITH, D. B. 1982. A computer model of speciation by founder effects. Genet Res., 39, 227-236.

COCKERHAM, C. C. 1967. Group inbreeding and coancestry. Genetics, 39, 89-104.

COCKerham, C. C. AND TACHIDA, H. 1988. Permanency of response to selection for quantitative characters in finite populations. Proc. Natl. Acad. Sci., U.S.A., 84, 62056209.

COCKERHAM, C. C. AND WEIR. B. s. 1973. Descent measures for two loci with some applications. Theoret. Pop. Biol., 4, 300-330.

COCKERHAM, C. C. AND WEIR, B. S. 1983. Variance of actual inbreeding. Theoret. Pop. Biol., 23, 85-109.

COHAN, F. M., HOFFMANN, A. A. AND GAYLEY, T. W. 1989. A test of the role of epistasis in divergence under uniform selection. Evolution, 43, 766-774.

CROW, J. F. AND KIMURA, M. 1970. An Introduction to Population Genetics Theory. Harper and Row, New York.

EFRON, B. 1982. The Jacknife, the Bootstrap and Other Resampling Plans. Society of Industrial and Applied Mathematics, Philadelphia, PA.

EFRON, B. AND GONG, G. 1983. A leisurely look at the bootstrap, the jackknife and cross-validation. Am. Sci., 37, 36-48.

FALCONER, D. s. 1989. Introduction to Quantitative Genetics. Longman, New York.

FISHER, R. A. 1958. The Genetical Theory of Natural Selection. Dover Publications, New York.

GEIGER, H. H. 1988. Epistasis and heterosis. In: Weir, B. S., Eisen, E. J., Goodman, M. M. and Namkoong, G. (eds) Proceedings of the Second International Conference on Quantitative Genetics, Sinauer Associates, Sunderland, MA, pp. 395-399.

GooDNIGHT, C. J. 1987. On the effect of founder events on epistatic genetic variance. Evolution, 41, 171-179.

GOODNIGHT, C. J. 1988. Epistasis and the effect of founder events on the additive genetic variance. Evolution, 42 , 441-454.

JAING, C. AND COCKERHAM, C. C. 1990. Quantitative genetic components within restricted populations. Crop Sci., 30, 7-12.

KEARSEY, M. J. AND KOJMA, K. 1967. The genetic architecture of body weight and egg hatchability in Drosophila melanogaster. Genetics, 56, 23-37.

LANDE, R. 1976. The maintenance of genetic variability by mutation in a polygenic character with linked loci. Genet. Res., Camb., 26, 221-235.

LANDE, R. 1980. Genetic variation and phenotypic evolution during allopatric speciation. Am. Nat., 116, 463-479.

LINTS, F. A. AND BOURGoIs, M. 1982. A test of the genetic revolution hypothesis of speciation. In: Lakovaara, S. (ed.) Advances in Genetics, Development and Evolution of Drosophila, Plenum, New York.

LOPEZ-FANJUL, C. AND VILLAVERDE, A. 1989. Inbreeding increases genetic variance for viability in Drosophila melanogaster. Evolution, 43, 1800-1804.

LYNCH, M. 1985. The rate of polygenic mutation. Genet. Res., Camb., 51, 137-148.

LYNCH, M. 1988. Design and analysis of experiments on random drift and inbreeding depression. Genetics, 120, 791-807.

McCOMMAS, S. A. AND BRYANT, E. H. 1990. Loss of electrophoretic variation in serially bottlenecked populations. Heredity, 64, 315-321.

MEFFERT, L. M. AND BRYANT, E. H. 1991. Mating propensity and courtship behavior in serially bottlenecked lines of the housefly. Evolution, 45, 293-306.

PATERSON, A. H., DeVERNA, J. W., LANINI, B. AND TANSKSLEY, S. D. 1990. Fine mapping of quantitative trait loci using selected overlapping recombinant chromosomes, in an interspecies cross of tomato. Genetics, 124, 735-742.

POLANS, N. O. AND ALLARD, R. w. 1989. An experimental evaluation of the recovery potential of ryegrass populations from genetic stress resulting from restriction of population size. Evolution, 43, 1320-1324.

ROBERTSON, A. 1952. The effect of inbreeding on the variation due to recessive genes. Genetics, 37, 189-207.

ROBERTSON, A. 1955. Selection in animals: synthesis. Cold. Spr. Harbr. Symp. Quant. Biol., 20, 225-229.

SHRIMPTON, A. E. AND ROBERTSON, A. 1988a. The isolation of polygenic factors controlling bristle score in Drosophila melanogaster. I. Allocation of third chromosome sternopleural bristle effects to chromosomal sections. Genetics, 118, 437-443.

SHRIMPTON, A. E. AND ROBERTSON, A. 1988b. Isolation of polygenic factors controlling bristle score in Drosophila melanogaster. II. Distribution of third chromosome bristle effects within chromosome sections. Genetics, 118, 445-459.

TACHIDA, H. AND COCKERHAM, C. c. 1989a. Effects of identity disequilibrium and linkage on quantitative variation in finite populations. Genet. Res., 53, 63-70.

TACHIDA, H. AND COCKERHAM, C. C. 1989 b. A building block model for quantitative genetics. Genetics, 121, 839-844.

WEIR, B. S. AND COCKERHAM, C. C. 1973. Mixed selfed and random mating at two loci. Genet. Res., 21, 247-262.

WRIGHT, s. 1951. The genetical structure of populations. Ann. Eugen., 15, 323-354.

WRIGHT, s. 1965. Factor interaction and linkage in evolution. Proc. R. Soc. B., 162, 80-104.

WRIGHT, s. 1969. Evolution and the Genetics of Populations, Vol. 2. University of Chicago Press, Chicago, IL.

WRIGHT, s. 1977. Evolution and the Genetics of Populations, Vol. 3. University of Chicago Press, Chicago, $\amalg$. 